\title{
Mammakarzinom: Genetischer Risikotest kann die Chemotherapie ersparen
}

\section{Der 21-Gen-Rezidivscore (RS) Oncotype DX ist als prognostischer/prädiktiver Test beim frühen Hormonrezeptor(HR)-positiven Brustkrebs validiert. Jetzt liegen 3-Jahres-Daten für Patientinnen vor, die auf Basis des Testergebnisses nur eine adjuvante endokrine Therapie erhalten haben.}

m Rahmen der Studie PlanB wurde prospektiv eine zentrale Tumorbank mit Mammakarzinomen (mittleres bis hohes Risiko, HER2-negativ) aufgebaut. Nach einer Zwischenauswertung wurde empfohlen, bei Patientinnen mit HR-positivem, pN0/1-Tumor und einem Risiko-Score (RS) von $\leq 11$ auf eine adjuvante Chemotherapie zu verzichten.

Zwischen 2009 und 2011 wurden im Rahmen von PlanB 3.198 Patientinnen (median 56 Jahre) behandelt. $41,1 \%$ wiesen eine nodal positive, $32,5 \%$ eine Grad-3-Erkrankung auf. Wegen eines RS-Werts von $\leq 11$ erhielten 348 Patientinnen $(15,3 \%)$ keine Chemotherapie. Nach median 35 Monaten betrug die 3-Jahres-Rate für das krankheitsfreie Überleben (DFS) bei diesen Frauen $98 \%$, bei Patientinnen mit einem RS $>25$ mit Chemotherapie $92 \%$ und bei Patientinnen mit RS $\geq 12$ und $\leq 25$ mit Chemotherapie $98 \%$.

In der multivariaten Analyse erwiesen sich der Nodalstatus, das Grading (zentral und lokal bestimmt) und der RSWert als unabhängige prognostische Faktoren. Lokale und zentrale Histologie waren bezüglich des Tumorgradings in $44 \%$ der Fälle diskordant. Der RS war moderat positiv mit dem Ki67-Wert und dem Tumorgrad und negativ mit PRund ER-Status korreliert.

Fazit: Das 3-Jahres-DFS der Patientinnen, die wegen eines $\mathrm{RS} \leq 11$ keine Chemotherapie erhalten hatten, war sehr gut. Nachdenklich stimmt die hohe Diskordanz des Gradings zwischen lokaler und zentraler Beurteilung und zwischen traditionellen prognostischen Markern und dem RS-Wert. Die Forscher schlagen bessere Standardisierung und Integration eines gut evaluierten Gentests wie des Oncotype DX zusätzlich zu klinischen und pathologischen Prognosefaktoren vor, um über eine Chemothera- pie bei frühem HR-positivem Brustkrebs zu entscheiden. Friederike Klein

Gluz O et al. West German Study Group Phase III PlanB Trial: First Prospective Outcome Data for the 21-Gene Recurrence Score Assay and Concordance of Prognostic Markers by Central and Local Pathology Assessment. J Clin Oncol. 2016;34(20):2341-9.

\section{Kommentar von Prof. Janni und Herrn Polasik:}

„Nicht jede Patientin mit frühem HR-positivem Mammakarzinom benötigt eine adjuvante Chemotherapie." Diese Aussage wird durch die hier vorgestellten Ergebnisse der PlanB-Studie unterstrichen. Dadurch steigen die Erwartungen an prädiktive Genxpressionstests wie den Oncotype-DX nun noch mehr. In diesem Test wird Tumorgewebe bzgl. der Aktivität von 21 Genen untersucht, um mithilfe des generierten RS eine Aussage über das Rezidiv-Risiko und somit die Notwendigkeit einer adjuvanten Chemotherapie zu treffen [Sinn P et al. Geburtsh Frauenheilk. 2013;73(9):932-40].

Bereits im Jahr 2004 untersuchten Soonmyung Paik und Kollegen im Rahmen der NSABP B-14-Studie, ob die Ergebnisse eines Genexpressionstests mit dem Auftreten von Rezidiven bei Patientinnen mit nodal-negativem Brustkrebs unter Tamoxifen-Therapie korrelierten [Paik S et al. N Engl J Med. 2004; 351(27):2817-26]. In der multivariaten Analyse konnte eine signifikante Vorhersagekraft für den Brustkrebstest nachgewiesen werden, die unabhängig vom Alter der Patientin und der Tumorgröße ist. Eine weitere multivariate Analyse wies eine signifikant prognostische Vorhersagekraft für das Auftreten von Metastasen binnen 5 Jahren nach [Sgroi DC et al. Lancet Oncol. 2013;14(11):1067-76].

Diese Ergebnisse führten schließlich zur Aufnahme des Oncotype-DX in die Empfehlungen der Arbeitsgemeinschaft Gynäkologische Onkologie (Evidenzgrad 1).

Dass der Oncotype-DX im Zweifel zu Recht in die Entscheidung einbezogen werden sollte, ob bei dem entsprechenden Patientinnenkollektiv eine Chemotherapie nötig ist, bestätigen jetzt auch die Ergebnisse aus der PlanB-Studie nach einem Follow-up von 3 Jahren. Dieser Genexpressionstest ist jedoch nur ein Baustein im Entscheidungsprozess. In den vorgestellten Ergebnissen der multivariaten Analyse stellen neben dem RS auch Nodalstatus und Entartungsgrad voneinander unabhängige prognostische Faktoren dar. Kritisch hierbei ist die hohe Diskordanz beim Grading zwischen lokaler und zentraler Bewertung zu sehen, zumal das Grading ebenfalls einen hohen prognostischen Wert hat. An dieser Stelle ist die Forderung der Forscher nach einer besseren Standardisierung zu unterstreichen.

Zusammenfassend ist festzuhalten, dass personalisierte Therapiestrategien beim frühen wie beim metastasierten Mammakarzinom zukünftig dominierend sein werden. So wird die Vorhersage eines Genexpressionstests wie des Oncotype-DX bei

\section{"Nicht jede Patientin mit frühem HR-positivem Brustkrebs benötigt eine adjuvante Chemotherapie."}

Patientinnen mit früher Brustkrebserkrankung ebenso wichtig sein wie Auftreten und Phänotyp von zirkulierenden Markern, zirkulierenden Tumorzellen oder zirkulierender Tumor-DNA beim metastasierten Mammakarzinom. Ziel dieser Strategie ist es, ein möglichst auf die Patientin abgestimmtes und somit optimales Therapiekonzept zu erstellen, um neben einem verlängerten Gesamt- und progressionsfreien Überleben auch die Lebensqualität deutlich zu verbessern.

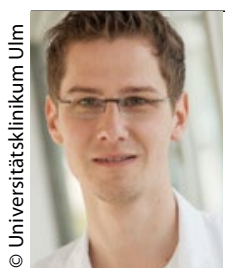

Arkadius Polasik Frauenklinik Universitätsklinikum UIm Arkadius.Polasik@ uniklinik-ulm.de

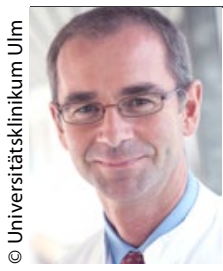

Prof. Dr. med. Wolfgang Janni Frauenklinik Universitätsklinikum UIm wolfgang.janni@ uniklinik-ulm.de 